\title{
Virtual reality and music therapy as distraction interventions to alleviate anxiety and improve mood states in breast cancer patients during chemotherapy
}

\author{
Andrea Chirico $^{1,2}$ | Patrizia Maiorano ${ }^{3}$ | Paola Indovina ${ }^{2,4}$ | Carla Milanese ${ }^{2,3} \mid$ \\ Giovan Giacomo Giordano $^{2}$ | Fabio Alivernini ${ }^{5}$ | Giovanni lodice ${ }^{6}$ | Luigi Gallo ${ }^{4}$ | \\ Giuseppe De Pietro ${ }^{4}$ (] | Fabio Lucidi ${ }^{1}$ | Gerardo Botti ${ }^{7}$ | Michelino De Laurentiis ${ }^{6}$ | \\ Antonio Giordano ${ }^{2,3}$
}

\author{
${ }^{1}$ Department of Social and Developmental \\ Psychology, "Sapienza" University of Rome, \\ Rome, Italy \\ ${ }^{2}$ Sbarro Institute for Cancer Research and \\ Molecular Medicine, Center for Biotechnology, \\ College of Science and Technology, Temple \\ University, Philadelphia, Pennsylvania \\ ${ }^{3}$ Department of Medical Biotechnologies, \\ University of Siena, Siena, Italy \\ ${ }^{4}$ Institute for High Performance Computing \\ and Networking, National Research Council of \\ Italy (ICAR-CNR), Naples, Italy \\ ${ }^{5}$ National Institute for the Evaluation of the \\ Education System (INVALSI), Rome, Italy \\ ${ }^{6}$ Breast Oncology Division, Istituto Nazionale \\ Tumori IRCCS Fondazione G. Pascale, \\ Napoli, Italy \\ ${ }^{7}$ Pathology Division, Istituto Nazionale Tumori \\ IRCCS Fondazione G. Pascale, Napoli, Italy

\section{Correspondence} \\ Antonio Giordano, Sbarro Institute for Cancer \\ Research and Molecular Medicine, Center for \\ Biotechnology, College of Science and \\ Technology, Temple University, 1900N 12th \\ St., Philadelphia, PA 19122. \\ Email: president@shro.org \\ Funding information \\ Sbarro Health Research Organization \\ (shro.org); Commonwealth of Pennsylvania
}

\begin{abstract}
Psychological distress is a common consequence of breast cancer diagnosis and treatment and could further exacerbate therapy side effects. Interventions increasing treatment tolerance are crucial to improve both patients' quality of life and adherence to therapies. Virtual reality (VR) has emerged as an effective distraction tool for different medical procedures. Here, we assessed the efficacy of immersive and interactive VR in alleviating chemotherapy-related psychological distress in a cohort of Italian breast cancer patients, also comparing its effects with those of music therapy (MT). Thirty patients were included in the VR group, 30 in the MT group, and 34 in the control group, consisting of patients receiving standard care during chemotherapy. Our data suggest that both VR and MT are useful interventions for alleviating anxiety and for improving mood states in breast cancer patients during chemotherapy. Moreover, VR seems more effective than MT in relieving anxiety, depression, and fatigue.
\end{abstract}

KEYWORDS

anxiety, breast cancer, chemotherapy, cybersickness, mood states, music therapy, virtual reality

\section{1 | INTRODUCTION}

Breast cancer, the most common malignancy in women worldwide, severely affects both physical and psychological health. Psychological distress is a consequence of breast cancer diagnosis (Chirico, Lucidi, Mallia, D'Aiuto, \& Merluzzi, 2015), with anxiety and depression being frequent manifestations (Hill et al., 2011).

Among the standard treatments for breast cancer, chemotherapy has several side effects, which lead to a further decline in quality of life (Mills, Parker, Dimsdale, Sadler, \& Ancoli-Israel, 2005). Moreover, psychological distress can increase the risk of developing chemotherapy side effects 
(Grassi et al., 2015), which in turn might trigger the development of conditioned responses to this treatment, such as anticipatory nausea (Kamen et al., 2014; Schneider, Kisby, \& Flint, 2011). Severe adverse effects can lead to dose decrease or treatment discontinuation. Therefore, early interventions able to improve treatment tolerance could be crucial to increase the chances of recovery. Over the last decades, many trials assessing psychological interventions have been conducted in patients with cancer, mainly in women with breast cancer (Lutgendorf, Sood, \& Antoni, 2010). Importantly, it has been suggested that breast cancer patients receiving psychological interventions were more likely to adhere to their chemotherapy regimen (Andersen et al., 2004).

Among psychological interventions aimed to improve tolerance to chemotherapy, distraction could be a promising strategy. Indeed, according to the Lazarus and Folkman's stress and coping model, when individuals recognize that nothing can be done to change a stressful condition, they regulate the emotional response to this condition through emotion-focused coping strategies (Lazarus \& Folkman, 1984). These strategies include avoidance, minimization, distancing, selective attention, making positive comparisons, and finding positive value in negative events. Distraction is an effective emotion-focused coping strategy because it diverts attention from unpleasant stimuli towards pleasant or interesting stimuli, thus decreasing stress and anxiety (Schneider \& Hood, 2007). Several distraction interventions, such as progressive relaxation, guided imagery, and cognitive distraction (reading, humor, music, and movies), have shown some success in patients undergoing chemotherapy (Schneider \& Hood, 2007; Schneider et al., 2011).

A type of distraction intervention able to alleviate distress and treatment-related symptoms is receptive music therapy (MT), which includes any intervention in which the patient listens to music with the aid of a reproducer under the guidance of the therapist (Atiwannapat, Thaipisuttikul, Poopityastaporn, \& Katekaew, 2016; Bruscia, 2018). Overall, evidence from a recent literature review on cancer patients receiving MT, supports a positive effect on anxiety, depression, pain, and quality of life (Gramaglia et al., 2019). Specifically, in patients undergoing chemotherapy, MT seems to reduce anxiety, tension, depression, and anger. A meta-analysis also indicated the effectiveness of therapistprovided music interventions in reducing the severity of anxiety and depression, with moderate to large effects being observed in cancer patients (Tsai et al., 2014). Other observed effects of MT during chemotherapy include relaxation (Burrai, Micheluzzi, \& Bugani, 2014) and reduction of the treatment side effects (Moradian \& Howell, 2015).

Over the last two decades, virtual reality (VR), a technology that allows users to immerse themselves in a three-dimensional computergenerated world, has found a variety of applications in health care (Indovina et al., 2018; Napolitano et al., 2013) and, in particular, has shown efficacy as a distraction tool to alleviate pain and distress during medical procedures, including chemotherapy (Chirico, Lucidi, et al., 2016; Indovina et al., 2018). VR equipment usually includes head-mounted displays, motion tracking systems, headphones, and devices to interact with the virtual environment. A key factor underlying VR distraction power is its ability to engage different senses simultaneously, by providing synthetic stimuli, such as visual imagery, spatialized sound, and sometimes tactile and olfactory feedback. Indeed, the provision of these multimodal stimuli, together with the ability to sense the user's motion and modify the virtual world accordingly, induce a sense of "presence" in the virtual environment, which is an individual user response found to be correlated with VR efficacy (Indovina et al., 2018). Moreover, the VR equipment can visually isolate patients from the medical environment and help them to focus on pleasant stimuli, thus possibly reducing negative emotions and inducing, instead, emotions with a positive valence, which are deemed to influence VR efficacy (Schneider \& Hood, 2007; Sharar et al., 2016; Triberti, Repetto, \& Riva, 2014). Also, different studies showed that high-quality and interactive VR systems are more effective (Indovina et al., 2018). Thus, interactivity and immersion (which, unlike the subjective sense of presence, is the objective and measurable level of sensory fidelity provided by VR; Bowman \& McMahan, 2007) are crucial factors impacting on VR distraction efficacy.

In the present study, using the above described Lazarus and Folkman's stress and coping model as a theoretical framework, we assessed the efficacy of immersive and interactive VR as a distraction intervention aimed to relieve chemotherapy-related anxiety and negative mood states in a cohort of Italian breast cancer patients, also comparing its effects with those of MT. VR and MT efficacy was evaluated versus a control group, consisting of patients receiving standard care during chemotherapy.

\section{2 | METHODS}

\section{1 | Participants}

Ninety-four female patients with breast cancer were recruited (starting from 2016) at the National Cancer Institute-IRCCS "Fondazione G. Pascale," Naples, Italy. Inclusion criteria were: patients' age between 18 and 70 years, breast cancer diagnosis, patients having to receive chemotherapy (second infusion without any history of previous neoadjuvant or adjuvant chemotherapy treatment) with the same type of drug (Epirubicin, Cyclophosphamide). Patients with epilepsy, drug and/or alcohol addictions, metastasis, wearing glasses, or having ports were excluded from the study.

\section{2 | Study design and procedure}

This is an externally controlled trial in which patients were randomly assigned to the VR or MT group and were compared with a nonconcurrently recruited control group.

Eligible participants were identified by medical oncologists and nurses. Participants provided written informed consent and completed a first set of questionnaires while waiting for treatment initiation. An experienced psychologist was available for any doubt regarding the questionnaires. Following the completion of the questionnaires, patients received VR, MT, or standard care during chemotherapy. Each chemotherapy treatment lasted 45-90 min. 
For the chemotherapy treatment with VR, a research nurse, together with a trained psychologist, explained how to use the VR equipment and helped patients with putting on the headset. Patients used the VR system for 5-10 min to get accustomed to it, and then the nurse administered the chemotherapy. Patients were able to report possible adverse symptoms while receiving VR and used the equipment for 20 min during chemotherapy.

For the MT condition, an mp3 reader and headphones were provided to the patients after $5 \mathrm{~min}$ from the starting of the chemotherapy infusion. Patients listened for 20 min relaxing music, pretaped by an expert music therapist.

For the control conditions, nurses and medical care staff followed customary procedures. Patients were free to choose different activities during treatment, including conversation and reading.

After the different procedures, patients completed a second set of questionnaires.

The study was approved by the Independent Ethical Committee of the National Cancer Institute-IRCCS "Fondazione G. Pascale" (Prot. n. "CEI 52/14").

\subsection{VR equipment and content}

The VR equipment consisted of head-mounted glasses (Vuzix Wrap 1200 VR) with a head motion tracking system. Each patient had a controller to interact with the virtual environment, which consisted of relaxing landscapes created on the Second Life ${ }^{\circledR}$ platform (Linden Lab). In particular, participants explored an island, by walking through a forest, observing different animals, climbing a mountain, and swimming in the sea.

\section{$2.4 \mid$ Measurements}

\subsection{1 | Patients' characteristics}

Patients' demographic characteristics were collected from standard self-report questionnaires. Clinical characteristics were obtained from the medical charts.

\subsection{2 | Anxiety measurement}

Patients' anxiety levels were measured using the State Anxiety Inventory (SAI) for adults (Spielberger, 1983), which is a valid and commonly used tool for measuring this emotion. SAI consists of 20 items, each scored on a 4-point Likert scale (1, almost never; 2 , occasionally; 3, most of the time; and 4, almost always). The SAI total scores range from 20 to 80 , with higher scores indicating higher levels of anxiety. SAI was used in this study because it evaluates current feelings of anxiety, rather than trait anxiety, through items that measure subjective feelings of apprehension, tension, nervousness, worry, and activation/arousal of the autonomic nervous system (Julian, 2011)

\subsection{3 | Measurement of mood states}

We used the short version of Profile of Mood States (SVPOMS; Shacham, 1983), which consists of the 37 items from the POMS that were found to have a higher internal consistency and were judged to be face-valid in different studies on cancer patients (Baker, Denniston, Zabora, Polland, \& Dudley, 2002). The SV-POMS maintains the six mood subscales from the POMS. Subscale scores are calculated according to the manual of the scale (DiLorenzo, Bovbjerg, Montgomery, Valdimarsdottir, \& Jacobsen, 1999). We used the Italian version of POMS (Mannarini, Polimeni, Shams, \& Giacobbo, 2012). The internal consistency reliabilities of POMS subscales range from 0.87 to 0.95 .

\subsection{4 | Evaluation of cybersickness symptoms}

The Virtual Reality Symptom Questionnaire (VRSQ), which was developed by Ames in 2005 (Ames, Wolffsohn, \& Mcbrien, 2005), was administered to patients after the VR intervention to evaluate the possible occurrence of symptoms of cybersickness, a type of motion sickness caused by exposure to VR. The questionnaire assesses eight general physical side effects (general discomfort, fatigue, boredom, drowsiness, headache, dizziness, concentration difficulties, and nausea) and five visual effects (tired eyes, aching eyes, eyestrain, blurred vision, and difficulties focusing) on a sevenpoint range (from 0 to 6 , with 0 indicating that the symptom is not present and 6 that the symptom is severe).

\section{5 | Data analysis}

Descriptive analyses were performed for all the variables of the study to check the distribution normality. A $2 \times 3$ repeated measures analysis of variance (ANOVA) with a two-level "within subjects" factor, namely TIME ("pretest" vs. "posttest"), and a three-level "between subjects" factor, that is INTERVENTION ("VR" vs. "MT" vs. "control group"), was performed using the SPSS software (version 25.0), considering as a dependent variable the perceived anxiety of the patients (as measured by SAI). A series of multivariate analysis of variances (MANOVAs) were performed considering as dependent variables all the subscales of the POMS and as independent variables the same as ANOVA. A descriptive analysis was performed for the cybersickness measure.

\section{3 | RESULTS}

\section{1 | Participants' characteristics}

A total sample of 94 subjects was assigned to the three groups of the study. In particular, 30 patients were included in the VR intervention 
TABLE 1 Baseline data of participants by groups

\begin{tabular}{|c|c|c|c|c|c|c|}
\hline Variables & $\begin{array}{l}\text { VR } \\
\text { group }\end{array}$ & $\%$ & $\begin{array}{l}\text { MT } \\
\text { group }\end{array}$ & $\%$ & $\begin{array}{l}\text { CG } \\
\text { group }\end{array}$ & $\%$ \\
\hline Age: mean $(S D)$ & $\begin{array}{r}55.18 \\
(5.7)\end{array}$ & & $\begin{array}{l}55.7 \\
(5.26)\end{array}$ & & $\begin{array}{l}56.2 \\
(6.79)\end{array}$ & \\
\hline $\begin{array}{l}\text { Marital status } \\
\text { Married } \\
\text { Single/widow/ } \\
\text { divorced }\end{array}$ & $\begin{array}{l}N=20 \\
N=10\end{array}$ & $\begin{array}{l}66.6 \\
33.3\end{array}$ & $\begin{array}{l}N=23 \\
N=7\end{array}$ & $\begin{array}{l}76.6 \\
23.3\end{array}$ & $\begin{array}{l}N=25 \\
N=9\end{array}$ & $\begin{array}{l}73.5 \\
26.4\end{array}$ \\
\hline $\begin{array}{l}\text { Employment } \\
\text { Yes } \\
\text { No }\end{array}$ & $\begin{array}{l}N=15 \\
N=15\end{array}$ & $\begin{array}{l}50 \\
50\end{array}$ & $\begin{array}{l}N=14 \\
N=16\end{array}$ & $\begin{array}{l}46.6 \\
53.3\end{array}$ & $\begin{array}{l}N=18 \\
N=16\end{array}$ & $\begin{array}{l}52.9 \\
47.0\end{array}$ \\
\hline $\begin{array}{l}\text { Tumor stage } \\
\text { I } \\
\text { II } \\
\text { III }\end{array}$ & $\begin{array}{l}N=6 \\
N=12 \\
N=12\end{array}$ & $\begin{array}{l}20 \\
40 \\
40\end{array}$ & $\begin{array}{l}N=8 \\
N=13 \\
N=9\end{array}$ & $\begin{array}{l}26.6 \\
43.3 \\
30\end{array}$ & $\begin{array}{l}N=9 \\
N=14 \\
N=11\end{array}$ & $\begin{array}{l}26.4 \\
41.1 \\
32.3\end{array}$ \\
\hline
\end{tabular}

Abbreviations: CG, control group; MT, music therapy; VR, virtual reality.

group, 30 patients in the MT intervention group, and 34 patients constituted the control group. Two participants in the VR group were too ill to complete the study questionnaire after the chemotherapy and therefore were withdrawn by the researcher. Data analysis included results from patients who completed all the questionnaires: $n=28$ for the VR group, $n=30$ for the MT group, and $n=34$ for the control group. Participants' sociodemographic characteristics and clinical stage are reported in Table 1. Statistical analysis of these variables did not show any significant difference among the three groups.

\section{2 | Anxiety}

A $2 \times 3$ analysis of variance (ANOVA) was performed, considering as a dependent variable the level of anxiety (as measured by SAI) and as independent variables a "within subjects" factor, namely TIME ("pretest" vs. "posttest"), and a "between subjects" factor, namely INTERVENTION ("VR" vs. "MT" vs. "control group"). Overall, the results of the ANOVA showed a significant interaction effect between the TIME factor and the INTERVENTION factor (TIME*INTERVENTION) on the patients' level of anxiety $(p=.006$; see Table 2). Furthermore, the analysis showed a significant main effect for the TIME factor only $(p<.001)$. Conversely, a nonsignificant main effect resulted from the ANOVA for the INTERVENTION factor $(p=.150)$.

Pairwise comparisons showed significant reductions in the anxiety levels between the pretest and the posttest for both the intervention groups (VR and MT) and a not significant reduction for the control group (Table 3). Moreover, pairwise comparisons did not show any significant difference among the three groups before the intervention, whereas significant differences between the VR and the control group ( $p=.001)$ and between the MT and the control group $(p=.049)$ were found after the procedure. Figure 1 reports the anxiety levels in the three groups, between the two times of data collection.

\section{3 | Mood states}

A $2 \times 2 \times 2$ mixed MANOVA was performed considering as dependent variables the levels of the six mood or affective states, as measured by the SV-POMS (i.e. tension, depression, anger, vigor, fatigue, and confusion), and as independent variables the same as reported above for the ANOVA test. Results of the univariate effects from MANOVA are reported in Table 4.

An interaction between the TIME and the INTERVENTION factors emerged for all the POMS subscales, except for vigor and confusion. Pairwise comparison analysis showed that patients participating in the VR intervention reported a significant reduction between the preintervention and the postintervention phases in the following negative mood states: tension, depression, anger, fatigue; similarly, patients receiving MT reported significantly reduced levels of tension, anger, and fatigue. Conversely, patients in the control group did not show any significant change in their mood states (Table 5). Moreover, pairwise comparisons showed significant differences among the three groups before the intervention on some variables of the POMS: the subjects in the VR group experienced more tension with respect to the subjects in the MT group $(p<.05)$; the VR group reported a higher level of anger than the control group $(p<.05)$; all the three groups differed significantly for fatigue (VR vs. MT: $p<.05$; VR vs. control group: $p<.001$; MT vs. control group $p<.05)$, with the VR group reporting the highest level of fatigue, followed by the MT group and the control group. Significant differences between the VR and the control group $(p<.001)$ and between the MT and the control group $(p<.05)$ were found after the procedure for depression, whereas tension was found to be significantly different after the procedure between the MT and the control group only $(p<.05)$. Figure 2 reports the mood state levels for which a significant interaction effect has been found in the three groups, between the two times of data collection. Furthermore, the analysis showed a significant main effect for the "within subjects" factor TIME on the following subscales of the POMS: tension, anger, and fatigue $(p<.001)$; also, a significant main effect resulted from the

TABLE 2 Univariate effects for the ANOVA analysis

\begin{tabular}{llllrr} 
Dependent variable & Effects & DF & $\boldsymbol{F}$ & $\boldsymbol{p}$ Value \\
\hline Anxiety & INTERVENTION & 2 & 1.959 & .147 & 0.042 \\
& TIME & 1 & 33.918 & $<.001$ \\
\hline
\end{tabular}


TABLE 3 Descriptive statistics for anxiety levels (as measured by the State Anxiety Inventory) for each group by the TIME factor, and indication of significant differences from pairwise comparisons

\begin{tabular}{|c|c|c|c|c|c|c|}
\hline INTERVENTION & TIME & Mean & $S D$ & \multicolumn{2}{|l|}{$95 \% \mathrm{Cl}$} & $\begin{array}{l}\text { Comparison } \\
p \text { value }\end{array}$ \\
\hline VR & Pre & 44.296 & 1.808 & 40.703 & 47.89 & $<.001$ \\
\hline \multirow[t]{2}{*}{ MT } & Pre & 42.9 & 1.715 & 39.491 & 46.309 & \multirow[t]{2}{*}{$<.001$} \\
\hline & Post & 39.567 & 1.597 & 36.392 & 42.741 & \\
\hline
\end{tabular}

Abbreviations: CG, control group; MT, music therapy; VR, virtual reality.

ANOVA for the INTERVENTION factor ("between subjects") on the tension and depression subscales $(p<.05)$.

\section{4 | Cybersickness symptoms}

Possible VR-associated cybersickness symptoms were analyzed through the VRSQ, which was administered to patients after the VR intervention. Data from this questionnaire showed that all symptoms, except a slight difficulty in concentrating, occurred with a frequency lower than $20 \%$ (see Table 6).

\section{4 | DISCUSSION}

Chemotherapy is a mainstay of cancer treatment; however, its side effects, such as nausea, vomiting, fatigue, pain, and anorexia, represent a major clinical problem. Moreover, psychological distress is a frequent consequence of cancer diagnosis and treatment (Derogatis et al., 1983; Stefanek, Derogatis, \& Shaw, 1987) and can further increase the risk of developing chemotherapy side effects

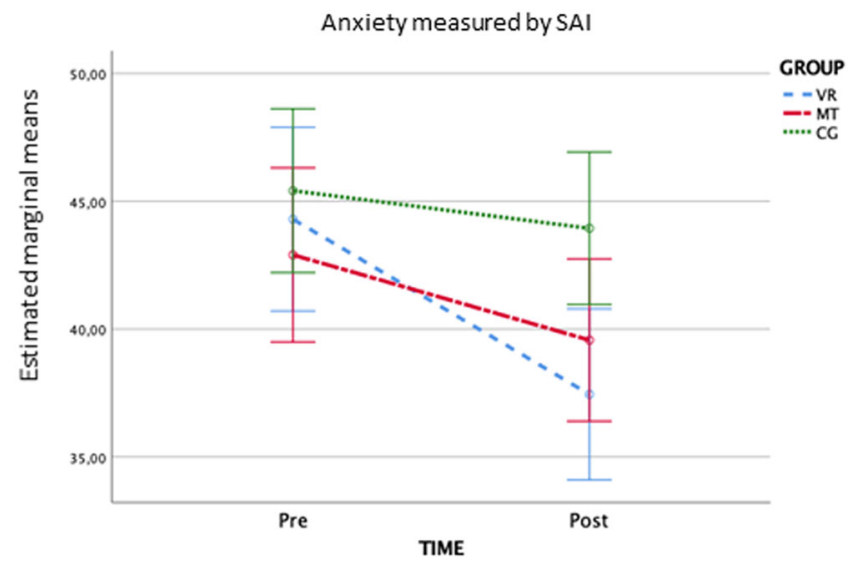

FIGURE 1 Line graph reporting the means with standard deviations of anxiety levels, as measured by the State Anxiety Inventory (SAI), in the virtual reality (VR) and music therapy (MT) intervention groups and in the control group (CG) between the two times of data collection
(Grassi et al., 2015). These adverse effects can result in reduced compliance to treatments. Therefore, the development of interventions able to enhance treatment tolerance is required to improve both patients' quality of life and chances of recovery.

Different distraction interventions have shown some success in patients receiving chemotherapy (Schneider \& Hood, 2007; Schneider et al., 2011). Among these interventions, VR, with its great attentiongrabbing power, proved to support patients with cancer in different settings (Bani Mohammad \& Ahmad, 2019; Chirico, Lucidi, et al., 2016), including during chemotherapy (Chirico, D'Aiuto, et al., 2016; Chirico, Lucidi, et al., 2016). Although these studies suggested the potential utility of VR to improve chemotherapy-related distress outcomes both in adults and in children with different cancer types (Chirico, Lucidi, et al., 2016; Indovina et al., 2018), most of them involved a very small number of patients (Oyama, Kaneda, Katsumata, Akechi, \& Ohsuga, 2000; Schneider \& Workman, 1999; Schneider, Ellis, Coombs, Shonkwiler, \& Folsom, 2003; Schneider, Prince-Paul, Allen, Silverman, \& Talaba, 2004) and the only study with a larger sample size did not achieve a statistically significant symptom improvement (Schneider \& Hood, 2007). Moreover, these trials used relatively low-tech-VR systems with barely interactive VR worlds and one study used a semi-immersive VR system (Oyama et al., 2000). Considering that the levels of immersion and interactivity impact on VR efficacy, stronger results might likely be obtained with more immersive and fully interactive VR worlds. Also, none of these studies compared the efficacy of VR with that of other forms of distraction in reducing chemotherapy-related symptoms.

This is the first study exploring the efficacy of immersive and interactive VR in relieving chemotherapy-related anxiety and negative mood states in a cohort of Italian breast cancer patients, also comparing its effects with those of MT. VR and MT efficacy was evaluated versus a control group, consisting of patients receiving standard care during chemotherapy, without any further supportive intervention. Approximately $78 \%$ of the participants were aged 50 years and above, consistent with the statistics on breast cancer by the Italian Association of Cancer Registries (AIRTUM), which reported that breast cancer mainly affects women of those ages (AIRTUM et al., 2016). We also analyzed motion sickness symptoms through a questionnaire (VRSQ) specifically developed for assessing possible VR-related side effects (Ames et al., 2005). All the assessed outcomes are discussed separately in the following sections. 
TABLE 4 Univariate effects for the multivariate analysis of variance (MANOVA), considering the subscales of the Profile of Mood States (POMS)

\begin{tabular}{|c|c|c|c|c|c|}
\hline Effects & Dependent variables & DF & $F$ & $p$ Value & $\eta_{\mathrm{p}}{ }^{2}$ \\
\hline \multirow[t]{5}{*}{ INTERVENTION } & Tension & 2 & 5.183 & .007 & 0.104 \\
\hline & Depression & 2 & 3.343 & .040 & 0.070 \\
\hline & Anger & 2 & 0.629 & .536 & 0.014 \\
\hline & Fatigue & 2 & 1.097 & .338 & 0.024 \\
\hline & Confusion & 2 & 1.959 & .147 & 0.042 \\
\hline \multirow{4}{*}{ TIME } & Anger & 1 & 24.205 & $<.001$ & 0.214 \\
\hline & Vigor & 1 & 1.373 & .244 & 0.015 \\
\hline & Fatigue & 1 & 25.190 & $<.001$ & 0.221 \\
\hline & Confusion & 1 & 2.299 & .133 & 0.025 \\
\hline \multirow[t]{2}{*}{ TIME * INTERVENTION } & Tension & 2 & 4.561 & .013 & 0.093 \\
\hline & Depression & 2 & 7.645 & .001 & 0.147 \\
\hline
\end{tabular}

\section{1 | Anxiety}

The significant interaction observed between the TIME factor ("pretest" vs. "posttest") and the INTERVENTION factor ("VR" vs. "MT" vs. "control group") on the patients' level of anxiety prompted us to perform pairwise comparison analyses.

We observed that all the three groups of patients had a high mean level of anxiety before chemotherapy (44.2 for VR; 42.9 for MT; 45.4 for control group, as measured by SAI), considering 40 as a clinical cut-off score (Knight, Waal Manning, \& Spears, 1983; Vodermaier, Linden, \& Siu, 2009). No significant differences in pretest anxiety were found among the three groups.

Although all the groups reported lower mean anxiety scores in the posttest than in the pretest, statistically significant differences were achieved only in the intervention groups (VR and MT), whereas the pre and posttest difference was not significant for the control group.

Notably, the VR group reported a significantly lower mean postintervention anxiety score compared with that of the control group $(p=.001)$, in line with a previous study reporting a significant decrease in anxiety in 16 breast cancer patients (aged 50-77 years) after chemotherapy treatment with VR (Schneider et al., 2003). Conversely, in another study involving 20 younger breast cancer patients (aged 27-55 years), the decrease in anxiety after the VR intervention did not reach statistical significance (Schneider et al., 2004).

We also observed a lower mean posttest anxiety score in the MT group with respect to the control group; however, this difference barely reached the level of significance $(p=.049)$. Moreover, mean posttest anxiety was lower in the VR group than in the MS group (37.4 and 39.6, respectively), although without reaching statistical significance.

\section{2 | Mood states}

We observed a significant interaction between the TIME and the INTERVENTION factors for all the POMS subscales, except for vigor and confusion. Pairwise comparison analysis showed that patients receiving the VR intervention had a significant reduction in their tension, depression, anger, and fatigue levels between the pretest and the posttest. Similar results were also observed in the MT group, except for depression, which did not decrease significantly in these patients. Conversely, the control group did not show any significant pretest-posttest change. These results are consistent with previous studies suggesting that immersive (Schneider et al., 2004) or semiimmersive (Oyama et al., 2000) VR systems can impact on fatigue in adult patients with different cancer types. Notably, although MT and VR were both able to induce a significant decrease in fatigue between the pretest and the posttest, the comparison of the pretest-posttest reductions between MT and VR showed a greater effect for the VR condition (pretest-posttest for VR: 4.321; pretest-posttest for MT: 1.767). The suggested greater effectiveness of VR in decreasing both depression and fatigue with respect to MT seems to reflect the different characteristics of the two interventions: MT is aimed to induce relaxation (Pelletier, 2004), whereas VR could induce arousal (Sharar et al., 2016).

\section{3 | Cybersickness}

Results from the VRSQ showed that all symptoms occurred with a frequency $<20 \%$, which has been considered negligible by the authors of the scale (Ames et al., 2005), except a slight difficulty in concentrating (21.4\%). In line with previous observations (Indovina et al., 2018), nausea was very infrequent and mild. 
TABLE 5 Descriptive statistics for the Profile of Mood States subscales, for each group by the TIME factor, and indication of significant differences from pairwise comparisons

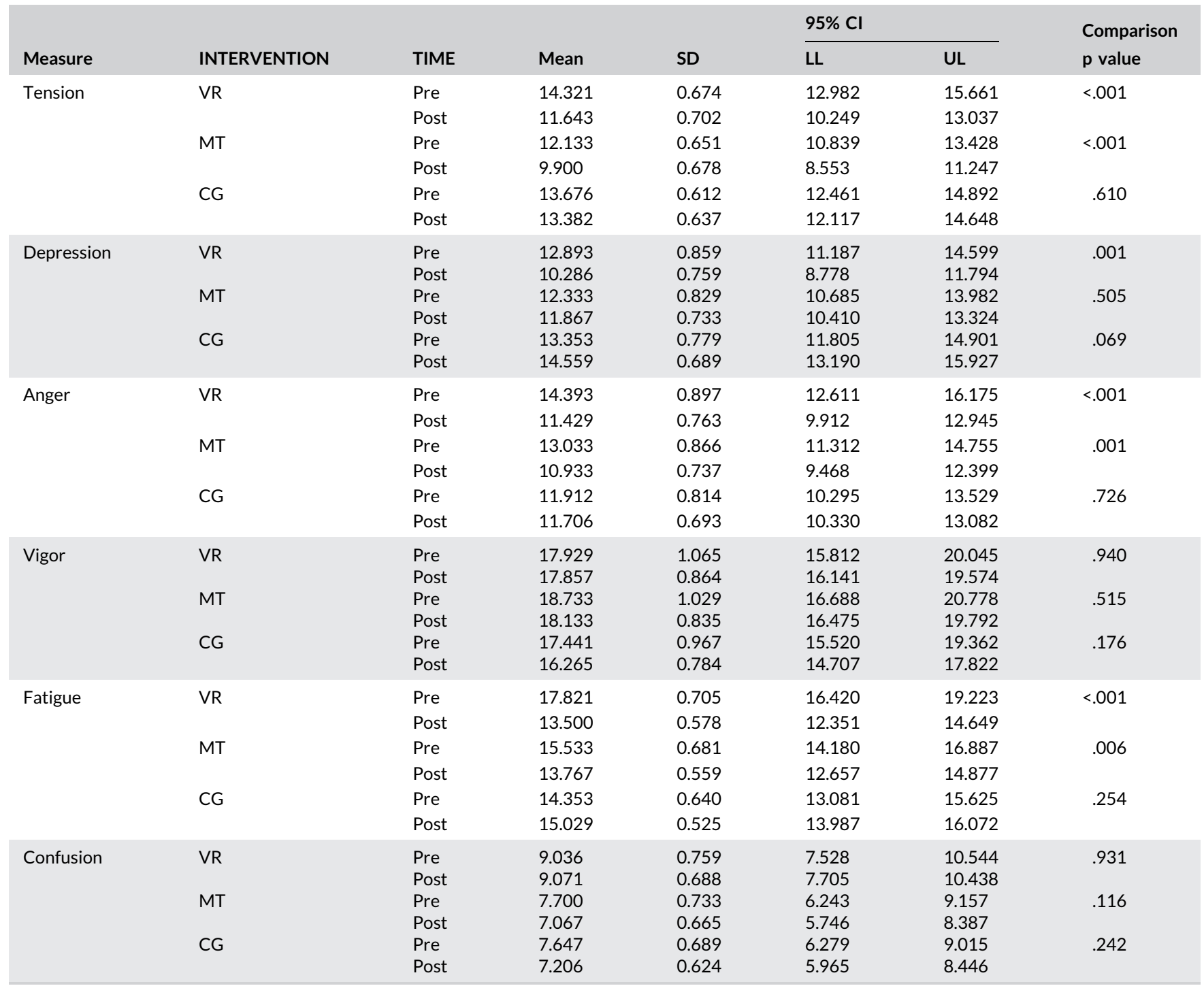

Abbreviations: VR, virtual reality; MT, music therapy; CG, control group.

Although occurring very infrequently, some symptoms were evaluated by the participants as severe. However, the symptoms were assessed only after the chemotherapy-VR intervention, without a comparison with the pretest conditions or with the control group, and therefore it is not possible to ascribe them entirely to the VR use. Indeed, at least some of these symptoms are common in cancer patients during chemotherapy. In particular, moderate-severe fatigue occurred in approximately $14 \%$ of patients; however, as reported above, VR proved to induce a decrease in fatigue (as measured by POMS) between the pretest and the posttest. Therefore, at least this symptom could not be ascribed to the VR intervention. It should also be considered that we used a relatively low-tech-VR system compared with those available in the market today. It is likely that the occurrence of cybersickness symptoms can be avoided by using state-of-the-art VR systems.

\section{5 | LIMITATIONS}

This study is unblinded and lacks a concurrently randomized control group and, therefore, is at risk of bias. Indeed, patients were randomly assigned to the VR or MT group and were compared with a separately recruited, nonrandomized control group.

Another limitation is that this trial analyzed only short-term effects. Indeed, measurements were taken only at two time points (preintervention and postintervention) and each patient participated in only one session. Further studies should explore potential longterm benefits of the VR intervention (e.g. a possible decrease in anticipatory anxiety before the subsequent chemotherapy sessions) and should also verify whether VR continues to be effective over repeated exposures. Indeed, a concern regarding the use of VR during medical procedures is that it could lose efficacy over several sessions since individuals might habituate to it. This remains to be 
Tension

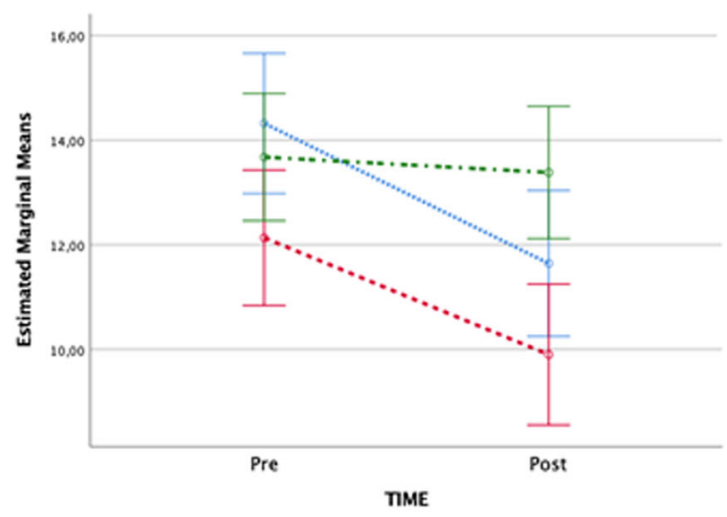

Anger

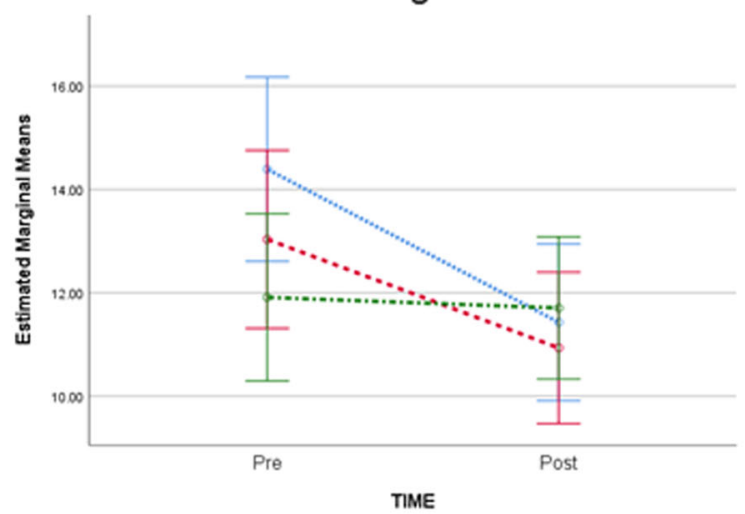

Depression

Group

$\cdots . .$. VR
$\cdots$ MT
$\cdots \cdot{ }_{C G}$

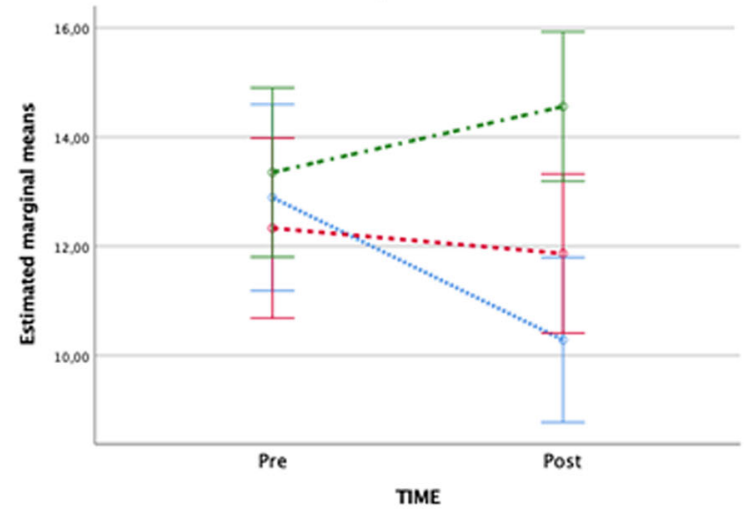

Fatigue

Group

$\cdots \cdot V R$
$\cdots \cdot M T$
$\cdots \cdot C G$

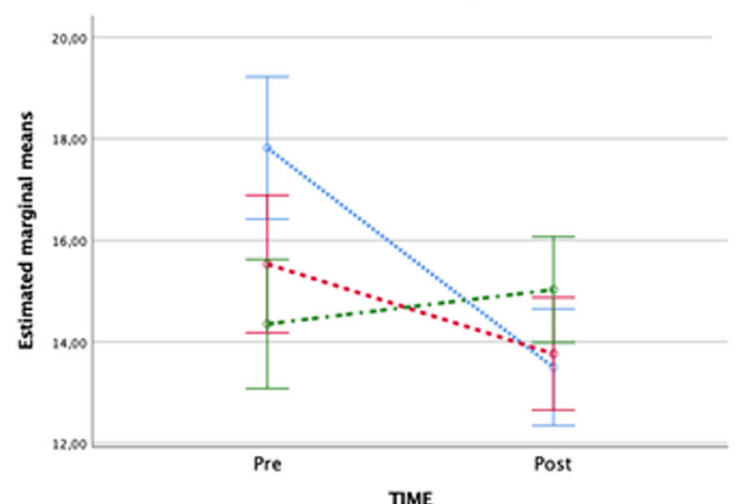

Group

…vV VR

-..CC

FIGURE 2 Line graph reporting the means with standard deviations of the tension, depression, anger, and fatigue levels, as measured by the Profile of Mood States (POMS) subscales, in the virtual reality (VR) and music therapy (MT) intervention groups and in the control group (CG) between the two times of data collection

TABLE 6 Percentage frequency of the occurrence of each symptom by the items of the Virtual Reality Symptom Questionnaire (VRSQ)

\begin{tabular}{|c|c|c|c|c|c|c|c|}
\hline \multirow[b]{2}{*}{ Symptom } & \multirow{2}{*}{$\begin{array}{l}\text { None } \\
0\end{array}$} & \multicolumn{2}{|c|}{ Slight } & \multicolumn{2}{|c|}{ Moderate } & \multicolumn{2}{|c|}{ Severe } \\
\hline & & 1 & 2 & 3 & 4 & 5 & 6 \\
\hline General discomfort & 82.1 & 3.6 & 7.1 & 0 & 7.1 & 7.1 & 0 \\
\hline Fatigue & 78.6 & 7.1 & 0 & 0 & 7.1 & 7.1 & 0 \\
\hline Boredom & 57.1 & 17.9 & 7.1 & 0 & 10.7 & 7.1 & 0 \\
\hline Drowsiness & 50.0 & 17.9 & 7.1 & 3.6 & 10.7 & 7.1 & 3.6 \\
\hline Headache & 67.9 & 10.7 & 10.7 & 0 & 3.6 & 3.6 & 3.6 \\
\hline Dizziness & 89.3 & 3.6 & 7.1 & 0 & 0 & 0 & 0 \\
\hline $\begin{array}{l}\text { DIfficulty } \\
\text { concentrating }\end{array}$ & 64.3 & 14.3 & 21.4 & 0 & 0 & 0 & 0 \\
\hline Nausea & 85.7 & 3.6 & 7.1 & 0 & 3.6 & 0 & 0 \\
\hline Tired eyes & 67.9 & 10.7 & 7.1 & 3.6 & 10.7 & 0 & 0 \\
\hline Aching eyes & 82.1 & 7.1 & 10.7 & 0 & 0 & 0 & 0 \\
\hline Eyestrain & 78.6 & 17.9 & 0 & 3.6 & 0 & 0 & 0 \\
\hline Blurred & 82.1 & 10.7 & 7.1 & 0 & 0 & 0 & 0 \\
\hline Difficulties focusing & 78.6 & 3.1 & 2.1 & 0 & 0 & 0 & 3.6 \\
\hline
\end{tabular}

assessed, although some encouraging data showed that the benefits of the VR distraction intervention were not lost across at least three sessions, thus suggesting that VR effects are not ascribable only to the experience novelty (Indovina et al., 2018).

Moreover, only self-reported measurements were analyzed. Future studies evaluating the effects of VR on physiological variables (such as heart rate and skin conductance) or on stress-related molecules might provide a more comprehensive evaluation of distress (La Montagna et al., 2019).

Although our study population was larger than that of previous clinical trials on VR intervention during chemotherapy in breast cancer patients (Schneider et al., 2003, 2004), studies involving a greater number of patients are necessary to generalize the observations and also to evaluate possible associations between VR efficacy and patients' characteristics, such as age and ethnicity.

Furthermore, VR-related symptoms were assessed only following the chemotherapy-VR intervention and, therefore, part of these symptoms could be ascribed to the chemotherapy treatment or to the condition of the patients.

Finally, we used a low-tech-VR system with respect to the new generation of VR systems available today, which are more immersive and interactive. Therefore, these new systems could prove more 
effective in relieving chemotherapy-related distress, without inducing side effects.

\section{6 | CONCLUSIONS}

The results of our study suggest that both VR and MT are useful interventions for alleviating anxiety and for improving mood states in breast cancer patients during chemotherapy. Moreover, the VR intervention seems more effective than MT in relieving anxiety, depression, and fatigue. Thus, despite its limitations, this study supports the continuous research on VR as a distraction intervention able to meet the global clinical need for effective nonpharmacologic adjuncts.

\section{ACKNOWLEDGMENTS}

The study was supported by the Sbarro Health Research Organization (http://www.shro.org) and the Commonwealth of Pennsylvania.

\section{CONFLICT OF INTERESTS}

The authors declare that there are no conflict of interests.

\section{DATA AVAILABILITY STATEMENT}

The data sets used and/or analyzed during the current study are available from the corresponding author on reasonable request.

\section{ORCID}

Luigi Gallo (D) http://orcid.org/0000-0002-1281-404X

Giuseppe De Pietro (D) http://orcid.org/0000-0002-4675-5957

Antonio Giordano (ID) http://orcid.org/0000-0003-4353-5390

\section{REFERENCES}

AIRTUM, B. S., Buzzoni, C., Mallone, S., Trama, A., Castaing, M., ... Dal Maso, L. (2016). Italian cancer figures--Report 2015: The burden of rare cancers in Italy. Epidemiologia E Prevenzione, https://doi.org/10. 19191/EP16.1S2.P001.035

Ames, S. L., Wolffsohn, J. S., \& Mcbrien, N. A. (2005). The development of a symptom questionnaire for assessing virtual reality viewing using a head-mounted display. Optometry and Vision Science, 82(3), 168-176. https://doi.org/10.1097/01.OPX.0000156307.95086.6

Andersen, B. L., Farrar, W. B., Golden-Kreutz, D. M., Glaser, R., Emery, C. F., Crespin, T. R., ... Carson, W. E. (2004). Psychological, behavioral, and immune changes after a psychological intervention: A clinical trial. Journal of Clinical Oncology, 22(17), 3570-3580. https://doi.org/10.1200/JCO. 2004.06.030

Atiwannapat, P., Thaipisuttikul, P., Poopityastaporn, P., \& Katekaew, W. (2016). Active versus receptive group music therapy for major depressive
disorder-A pilot study. Complementary Therapies in Medicine, 26, 141-145. https://doi.org/10.1016/j.ctim.2016.03.015

Baker, F., Denniston, M., Zabora, J., Polland, A., \& Dudley, W. N. (2002). A POMS short form for cancer patients: Psychometric and structural evaluation. Psycho-Oncology, 11(4), 273-281. https://doi.org/10.1002/ pon.564

Bani Mohammad, E., \& Ahmad, M. (2019). Virtual reality as a distraction technique for pain and anxiety among patients with breast cancer: $A$ randomized control trial. Palliative and Supportive Care, 17(1), 29-34. https://doi.org/10.1017/S1478951518000639

Bowman, D. A., \& McMahan, R. P. (2007). Virtual reality: How much immersion is enough? Computer, 40(7), 36-43. https://doi.org/10. 1109/MC.2007.257

Bruscia, K. (2018). A working definition of music therapy (3rd ed.). May. Barcelona Publisher, Retrieved at https://www.barcelonapublishers. com/defining-music-therapy-3rd-edition

Burrai, F., Micheluzzi, V., \& Bugani, V. (2014). Effects of live sax music on various physiological parameters, pain level, and mood level in cancer patients: A randomized controlled trial. Holistic Nursing Practice, 28(5), 301-311. https://doi.org/10.1097/HNP.0000000000000041

Chirico, A., D'Aiuto, M., Pinto, M., Milanese, C., Napoli, A., Avino, F., ... Lucidi, F. (2016). The elapsed time during a virtual reality treatment for stressful procedures. A pool analysis on breast cancer patients during chemotherapy. Smart Innovation, Systems and Technologies, 55, 731-738. https://doi.org/10.1007/978-3-319-39345-2_65

Chirico, A., Lucidi, F., De Laurentiis, M., Milanese, C., Napoli, A., \& Giordano, A. (2016). Virtual reality in health system: Beyond entertainment. A mini-review on the efficacy of VR during cancer treatment. Journal of Cellular Physiology, 231, 275-287. https://doi. org/10.1002/jcp.25117

Chirico, A., Lucidi, F., Mallia, L., D'Aiuto, M., \& Merluzzi, T. V. (2015). Indicators of distress in newly diagnosed breast cancer patients. PeerJ, 3(7):e1107. https://doi.org/10.7717/peerj.1107

Derogatis, L. R., Morrow, G. R., Fetting, J., Penman, D., Piasetsky, S., Schmale, A. M., ... Carnicke, C. L. M. (1983). The prevalence of psychiatric disorders among cancer patients. The Journal of the American Medical Association, 249(6), 751-757. https://doi.org/10. 1001/jama.1983.03330300035030

DiLorenzo, T. A., Bovbjerg, D. H., Montgomery, G. H., Valdimarsdottir, H., $\&$ Jacobsen, P. B. (1999). The application of a shortened version of the Profile of Mood States in a sample of breast cancer chemotherapy patients. British Journal of Health Psychology, 4(4), 315-325. https:// doi.org/10.1348/135910799168669

Gramaglia, C., Gambaro, E., Vecchi, C., Licandro, D., Raina, G., Pisani, C., ... Zeppegno, P. (2019). Outcomes of music therapy interventions in cancer patients-A review of the literature. Critical Reviews in Oncology/Hematology, 138, 241-254. https://doi.org/10.1016/j. critrevonc.2019.04.004

Grassi, L., Berardi, M. A., Ruffilli, F., Meggiolaro, E., Andritsch, E., Sirgo, A., ... Nanni, M. G. (2015). Role of psychosocial variables on chemotherapyinduced nausea and vomiting and health-related quality of life among cancer patients: A European study. Psychotherapy and Psychosomatics, 84(6), 339-347. https://doi.org/10.1159/000431256

Hill, J., Holcombe, C., Clark, L., Boothby, M. R. K., Hincks, A., Fisher, J., ... Salmon, P. (2011). Predictors of onset of depression and anxiety in the year after diagnosis of breast cancer. Psychological Medicine, 41(7), 1429-1436. https://doi.org/10.1017/S0033291710001868

Indovina, P., Barone, D., Gallo, L., Chirico, A., De Pietro, G., \& Giordano, A. (2018). Virtual reality as a distraction intervention to relieve pain and distress during medical procedures. Clinical Journal of Pain, 34, 858-877. https://doi.org/10.1097/AJP.0000000000000599

Julian, L. J. (2011). Measures of anxiety: State-Trait Anxiety Inventory (STAI), Beck Anxiety Inventory (BAI), and Hospital Anxiety and Depression Scale-Anxiety (HADS-A). Arthritis Care and Research, 63(Supp.1), https://doi.org/10.1002/acr.20561 
Kamen, C., Tejani, M. A., Chandwani, K., Janelsins, M., Peoples, A. R., Roscoe, J. A., \& Morrow, G. R. (2014). Anticipatory nausea and vomiting due to chemotherapy. European Journal of Pharmacology, 722(1), 172-179. https://doi.org/10.1016/j.ejphar.2013.09.071

Knight, R. G., Waal Manning, H. J., \& Spears, G. F. (1983). Some norms and reliability data for the State-Trait Anxiety Inventory and the Zung Self-Rating Depression scale. British Journal of Clinical Psychology, 22(4), 245-249. https://doi.org/10.1111/j.2044-8260. 1983.tb00610.x

Lazarus, R. S, \& Folkman, S. (1984). Stress, appraisal, and coping. In S. P. Company (Ed.), Behaviour research and therapy. https://doi.org/10. 1016/0005-7967(85)90087-7

La Montagna, R., Canonico, R., Alfano, L., Bucci, E., Boffo, S., Staiano, L., ... Giordano, A. (2019). Genomic analysis reveals association of specific SNPs with athletic performance and susceptibility to injuries in professional soccer players. Journal of Cellular Physiology, 235, 2139-2148. https://doi. org/10.1002/jcp.29118

Lutgendorf, S. K., Sood, A. K., \& Antoni, M. H. (2010). Host factors and cancer progression: Biobehavioral signaling pathways and interventions. Journal of Clinical Oncology, 28, 4094-4099. https://doi.org/10.1200/JCO.2009.26. 9357

Mannarini, S., Polimeni, S., Shams, M., \& Giacobbo, M. (2012). Assessing negative and positive mood states: The identification of a short form of the POMS scale in Italian oncology outpatients. Testing, Psychometrics, Methodology in Applied Psychology, 19(2), 135-145. https://doi.org/10.4473/TPM19.2.5

Mills, P. J., Parker, B., Dimsdale, J. E., Sadler, G. R., \& Ancoli-lsrael, S. (2005). The relationship between fatigue and quality of life and inflammation during anthracycline-based chemotherapy in breast cancer. Biological Psychology, 69(1 Spec. Iss.), 85-96. https://doi.org/10.1016/j.biopsycho. 2004.11.007

Moradian, S., \& Howell, D. (2015). Prevention and management of chemotherapy-induced nausea and vomiting. International Journal of Palliative Nursing, 21(5), 216-224. https://doi.org/10.12968/ijpn.2015.21. 5.216

Napolitano, M. A., Hayes, S., Russo, G., Muresu, D., Giordano, A., \& Foster, G. D. (2013). Using avatars to model weight loss behaviors: Participant attitudes and technology development. Journal of Diabetes Science and Technology, 7, 1057-1065. https://doi.org/10. 1177/193229681300700430

Oyama, H., Kaneda, M., Katsumata, N., Akechi, T., \& Ohsuga, M. (2000). Using the bedside wellness system during chemotherapy decreases fatigue and emesis in cancer patients. Journal of Medical Systems, 24(3), 173-182. https://doi.org/10.1023/ A:1005591626518

Pelletier, C. L. (2004). The effect of music on decreasing arousal due to stress: A meta-analysis. Journal of Music Therapy, 41(3), 192-214. https://doi.org/10.1093/jmt/41.3.192

Schneider, S. M., Ellis, M., Coombs, W. T., Shonkwiler, E. L., \& Folsom, L. C. (2003). Virtual reality intervention for older women with breast cancer. Cyberpsychology \& Behavior, 6(3), 301-307. https://doi.org/10. 1089/109493103322011605
Schneider, S. M., \& Hood, L. E. (2007). Virtual reality: A distraction intervention for chemotherapy. Oncology Nursing Forum, 34(1), 39-46. https://doi.org/10.1188/07.onf.39-46

Schneider, S. M., Kisby, C. K., \& Flint, E. P. (2011). Effect of virtual reality on time perception in patients receiving chemotherapy. Supportive Care in Cancer, 19(4), 555-564. https://doi.org/10. 1007/s00520-010-0852-7

Schneider, S. M., Prince-Paul, M., Allen, M. J., Silverman, P., \& Talaba, D. (2004). Virtual reality as a distraction intervention for women receiving chemotherapy. Oncology Nursing Forum, 31, 81-88.

Schneider, S. M., \& Workman, M. (1999). Effects of virtual reality on symptom distress in children receiving chemotherapy. Cyberpsychology \& Behavior, 2(2), 125-134. https://doi.org/10. 1089/cpb.1999.2.125

Shacham, S. (1983). A shortened version of the Profile of Mood States. Journal of Personality Assessment, 47(3), 305-306. https://doi.org/10. 1207/s15327752jpa4703_14

Sharar, S. R., Alamdari, A., Hoffer, C., Hoffman, H. G., Jensen, M. P., \& Patterson, D. R. (2016). Circumplex model of affect: A measure of pleasure and arousal during virtual reality distraction analgesia. Games For Health Journal, 5(3), 197-202. https://doi.org/10.1089/g4h.2015.0046

Spielberger, C. D. (1983). In C. D. Spielberger, R. L. Gorsuch, P. Vagg \& A. J. Gerard (Eds.), Manual for the state-trait anxiety inventory. Palo Alto: CA: Consulting Psychology Press.

Stefanek, M. E., Derogatis, L. P., \& Shaw, A. (1987). Psychological distress among oncology outpatients: Prevalence and severity as measured with the Brief Symptom Inventory. Psychosomatics, 28(10), 530-532. https://doi.org/10.1016/S0033-3182(87)72467-0

Triberti, S., Repetto, C., \& Riva, G. (2014). Psychological factors influencing the effectiveness of virtual reality-based analgesia: $A$ systematic review. Cyberpsychology, Behavior and Social Networking, 17(6), 335-345. https://doi.org/10.1089/cyber.2014.0054

Tsai, H. F., Chen, Y. R., Chung, M. H., Liao, Y. M., Chi, M. J., Chang, C. C., \& Chou, K. R. (2014). Effectiveness of music intervention in ameliorating cancer 'patients' anxiety, depression, pain, and fatigue: A meta-analysis. Cancer Nursing, 37(6), E35-E50. https:// doi.org/10.1097/NCC.0000000000000116

Vodermaier, A., Linden, W., \& Siu, C. (2009). Screening for emotional distress in cancer patients: A systematic review of assessment instruments. Journal of the National Cancer Institute, 101, 1464-1488. https://doi.org/10.1093/jnci/djp336

How to cite this article: Chirico A, Maiorano P, Indovina $\mathrm{P}$, et al. Virtual reality and music therapy as distraction interventions to alleviate anxiety and improve mood states in breast cancer patients during chemotherapy. J Cell Physiol. 2020;235:5353-5362. https://doi.org/10.1002/jcp.29422 